\title{
Plasma Levels, Temporal Trends and Clinical Associations between Biomarkers of Inflammation and Vascular Homeostasis after Pediatric Traumatic Brain Injury
}

\author{
Abhijit V. Lele ${ }^{a, b}$ Bhunyawee Alunpipatthanachai ${ }^{b}$ Qian Qiu ${ }^{a}$ \\ Crystalyn Clark-Bell $^{b}$ Arraya Watanitanon ${ }^{b}$ Anne Moore ${ }^{c}$ Randall M. Chesnut ${ }^{c}$ \\ William Armstead $^{d}$ Monica S. Vavilalaa, b \\ a Department of Anesthesiology and Pain Medicine, Harborview Injury Prevention and Research Center, \\ Harborview Medical Center, Seattle, WA, USA; ${ }^{b}$ Harborview Injury Prevention and Research Center, \\ University of Washington, Seattle, WA, USA; ${ }^{C}$ Department of Neurological Surgery and Orthopedics, \\ Harborview Medical Center, Seattle, WA, USA; ${ }^{\mathrm{d}}$ Department of Anethesiology and Critical Care, University of \\ Pennsylvania, Philadelphia, PA, USA
}

\section{Keywords}

Angiopoietin-2 · Endocan-1 · Endothelin-1 · Interleukin-6 • Children · Traumatic brain injury · Endothelium

\begin{abstract}
Expression of inflammatory (interleukin-6 [IL-6]) and vascular homeostatic (angiopoietin-2 [AP-2], endothelin-1 [ET-1], endocan-2 [EC-2]) biomarkers in pediatric traumatic brain injury (TBI) was examined in this prospective, observational cohort study of 28 children hospitalized with mild, moderate, and severe TBI by clinical measures (age, sex, Glasgow Coma Scale score [GCS], Injury Severity Score [ISS], and cerebral autoregulation status). Biomarker patterns suggest an inverse relationship between GCS and AP-2, GCS and IL-6, ISS and ET-1, but a direct relationship between GCS and ET-1
\end{abstract}

and ISS and AP-2. Biomarker patterns suggest an inverse relationship between AP-2 and ET-1, AP-2 and EC-2, but a direct relationship between AP-2 and IL-6, IL- 6 and EC-2, and IL- 6 and ET-1. Plasma concentrations of inflammatory and vascular homeostatic biomarkers suggest a role for inflammation and disruption of vascular homeostasis during the first 10 days across the severity spectrum of pediatric TBI. Although not statistically significant, without impact on cerebral autoregulation, biomarker patterns suggest a relationship between inflammation and alterations in vascular homeostasis. The large variation in biomarker levels within $\mathrm{TBI}$ severity and age groups, and by sex suggests other contributory factors to biomarker expression.

(c) 2019 S. Karger AG, Basel

This study was conducted at Harborview Medical Center.

\section{KARGER}

(c) 2019 S. Karger AG, Basel

karger@karger.com

www.karger.com/dne
Abhijit V. Lele, MBBS, MD, MS, FNCS

Department of Anesthesiology and Pain Medicine, Mail box

Harborview Medical Center, University of Washington

Seattle, WA 98104 (USA)

E-Mail abhijit2@uw.edu 


\section{Introduction}

Traumatic brain injury (TBI) is a leading cause of death and disability and affects $47-280$ of 100,000 children worldwide [1]. In the USA alone, an estimated 600,000 children under the age of 18 years are hospitalized, of whom 7,600 die, and another 5,000 are disabled each year $[2,3]$. Poor outcomes after TBI are associated with initial injury, as well as secondary injury from inflammation and vascular dysregulation [4]. Secondary brain injury consists of processes that are initiated in response to hypoxia, ischemia, or excitotoxic injury to neurons and astroglial cells, and biomarkers in plasma and cerebrospinal fluid may allow us to study these complex cascades after pediatric TBI [5-9]. However, biomarker expression of vascular homeostatic or inflammatory processes is not well characterized in children with TBI.

Angiopoietin-2 (AP-2) is a member of a family of vascular growth factors that plays a role in angiogenesis and in controlling microvascular permeability, vasodilation, and vasoconstriction by signaling vascular smooth muscle cells [10]. Increase in AP-2 promotes inflammation, and changes in AP-2 occur during illnesses $[11,12]$ including after cerebral ischemia [13, 14]. Angiopoietin also modulates blood-brain barrier breakdown and endothelial apoptosis [15], and the early phase of postinjury blood-brain barrier breakdown is associated with a reduction in angiopoietin-1 and upregulation of AP-2 [15]. However, patterns of expression after pediatric TBI have not been reported. Endothelin-1 (ET-1) is an endogenous vasoconstrictor peptide that plays a role in vascular homeostasis [16-18]. ET-1 is implicated in developmental outcomes of very low birth weight newborns with hypoxic encephalopathy [19], blast-induced TBI in rats [20], delayed cerebral hypoperfusion after global ischemia [21], and overexpression of ET-1 may contribute to dementia associated with ischemic stroke by exaggerating astrocyte-derived amyloid secretion [22], but its role in pediatric TBI is unknown. Endocan-2 (EC-2), also known as endothelial cell-specific molecule-1, may play a role during neoangiogenesis, and levels of endocan are increased in the presence of pro-angiogenic growth factors such as vascular endothelial growth factor or fibroblast growth factor. While endocan has been studied in cancer and pre-eclampsia $[23,24]$, there are no reports in TBI.

Cerebral inflammatory stimuli such as hypoxia and ischemia-reperfusion may stimulate biosynthesis of interleukins such as interleukin-6 (IL-6) in endothelium, glia, and leukocytes. Interleukins increase vascular permeability, modify the blood-brain barrier, and lead to

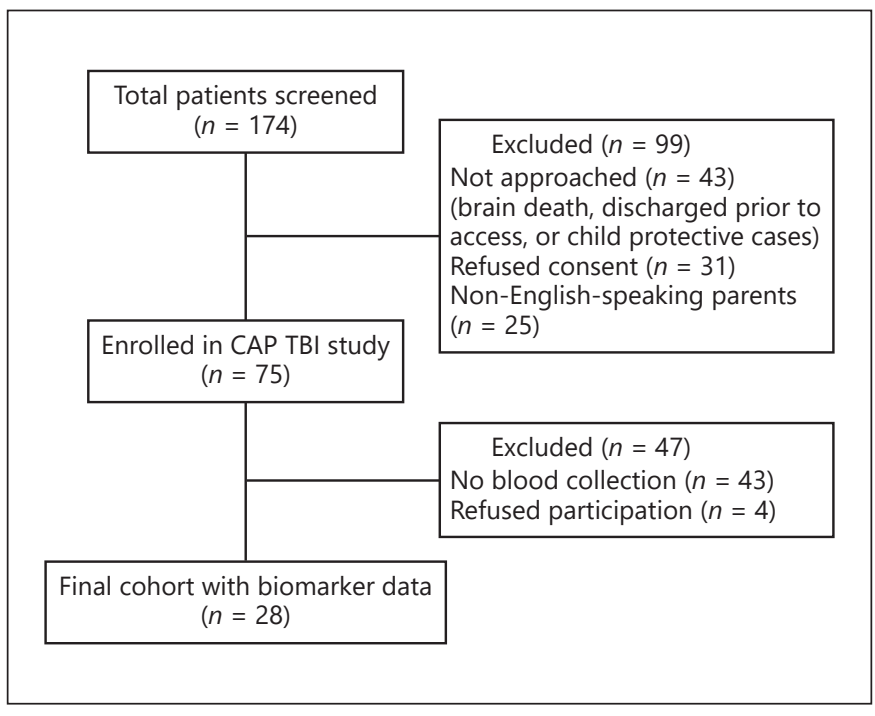

Fig. 1. Flow diagram for patient selection for biomarker assays after pediatric traumatic brain injury (TBI).

leukocyte accumulation. IL-6 activity has been implicated in preclinical and adult models of TBI [25-27] and may be expressed in the cerebrospinal fluid $[4,7,28,29]$ as well as the serum $[7,30,31]$. The expression of IL- 6 in the serum may decrease at the end of 1 week after TBI [31] and may be associated with both favorable [31] and unfavorable outcomes after pediatric TBI [7].

Given prior work in preclinical models of TBI [32] as well as the paucity of such information in pediatric TBI, we sought to understand the plasma levels, patterns, and relationships between inflammatory and vascular homeostatic biomarker expression in pediatric TBI.

\section{Methods}

Study Center and Data Sources

A prospective observational cohort study was performed on a convenience sample at Harborview Medical Center, which is a level I pediatric trauma center. This study was conducted between September 2016 and September 2018. Only hospitalized children under 18 years with a history of trauma, with evidence of TBI on computerized tomography (CT) of the head were eligible for inclusion. Pregnant minors, children under child protective services, patients with ongoing cardiopulmonary resuscitation, or patients with brain death were excluded per suggestion of the Institutional Review Board and anticipated challenges with obtaining blood samples. Research coordinators screened admission logs daily for eligible subjects. Informed consent was obtained from the patient's legally authorized representative, and assent was obtained from patients as appropriate and as soon as possible from the time of hospital admission (Fig. 1). Data from some patients in the cohort 
Table 1. Characteristics of children $(n=28)$ with mild* $(n=14)$, moderate** $(n=3)$ and severe ${ }^{* * *}(n=11)$ traumatic brain injury $(\mathrm{TBI})$

\begin{tabular}{|c|c|c|c|c|}
\hline Parameter & $\begin{array}{l}\text { All patients } \\
(n=28)\end{array}$ & $\begin{array}{l}\text { Mild TBI } \\
(n=14)\end{array}$ & $\begin{array}{l}\text { Moderate TBI } \\
(n=3)\end{array}$ & $\begin{array}{l}\text { Severe TBI } \\
(n=11)\end{array}$ \\
\hline Median age (IQR), years & $11(5-15.5)$ & $12(9-15)$ & $3(1-7)$ & $11(5-16)$ \\
\hline \multicolumn{5}{|l|}{ Age groups, $n(\%)$} \\
\hline $0-4$ years & $6(21.4)$ & $2(14.3)$ & $2(66.7)$ & $2(18.2)$ \\
\hline $5-9$ years & $6(21.4)$ & $2(14.3)$ & $1(33.3)$ & $3(27.3)$ \\
\hline $10-14$ years & $8(28.6)$ & $6(42.8)$ & 0 & $2(18.2)$ \\
\hline Males, $n(\%)$ & $18(64.3)$ & $9(64.3)$ & $2(66.7)$ & $7(63.7)$ \\
\hline Median ISS (IQR) & $25(17-27)$ & $17.5(11-26)$ & $41(26-41)$ & $21.5(17-27)$ \\
\hline Median admission GCS (IQR) & $13(6-15)$ & $15(14-15)$ & $9(9-9)$ & $4.5(3-6)$ \\
\hline \multicolumn{5}{|l|}{ Mechanism of injury, $n(\%)$} \\
\hline Motor vehicle crash & $10(35.7)$ & $2(14.3)$ & $1(33.3)$ & $7(63.6)$ \\
\hline Fall & $10(35.7)$ & $7(70)$ & 0 & $3(30)$ \\
\hline Skull fracture+ & $15(53.6)$ & $9(64.3)$ & $2(66.7)$ & $4(36.4)$ \\
\hline Isolated subgaleal hematoma & 0 & & & \\
\hline Subgaleal hematoma+ & $13(46.4)$ & $6(42.9$ & $1(33.3)$ & $6(53.6)$ \\
\hline Isolated subdural hematoma & $2(7.1)$ & $1(7.1)$ & $1(33.3)$ & \\
\hline Subdural hematoma+ & $12(42.9)$ & $8(57.1)$ & 0 & $4(36.4)$ \\
\hline Isolated subarachnoid hemorrhage & 0 & & & \\
\hline Subarachnoid hemorrhage+ & $12(42.9)$ & $6(42.9)$ & 0 & $6(54.6)$ \\
\hline Isolated epidural hematoma & $1(3.6)$ & & & $1(9.1)$ \\
\hline Epidural hematoma+ & $7(25)$ & $4(28.6)$ & $1(33.3)$ & $2(18.2)$ \\
\hline Isolated pneumocephalus & 0 & & & \\
\hline Pneumocephalus + & $7(25)$ & $3(21.4)$ & $2(66.7)$ & $2(18.2)$ \\
\hline Isolated intraparenchymal hemorrhage & $1(3.6)$ & $1(7.1)$ & & \\
\hline Isolated intraventricular hemorrhage & 0 & & & \\
\hline Intraventricular hemorrhage+ & $1(3.6)$ & 0 & 0 & $1(9.1)$ \\
\hline \multicolumn{5}{|l|}{ Median intensive care unit length of stay } \\
\hline (IQR), days & $1.9(1.1-5.6)$ & $1.6(1-1.8)$ & $4.1(1-5.8)$ & $4.6(2.1-10.3)$ \\
\hline \multicolumn{5}{|l|}{ Median hospital length of stay } \\
\hline (IQR), days & $6.5(1.7-11.5)$ & $1.8(1-5.5)$ & $8.7(7.1-13)$ & $10.9(7.5-16.1)$ \\
\hline Median discharge GCS (IQR) & $15(15-15)$ & $14.5(15-15)$ & $15(14-15)$ & $15(12-15)$ \\
\hline \multicolumn{5}{|l|}{ Discharge disposition, $n(\%)$} \\
\hline Home & $20(71.4)$ & $14(100)$ & $2(66.7)$ & $4(36.4)$ \\
\hline Inpatient rehabilitation facility & $7(25)$ & 0 & $1(33.3)$ & $6(54.5)$ \\
\hline Death & $1(3.6)$ & 0 & 0 & $1(9.1)$ \\
\hline
\end{tabular}

IQR, interquartile range; ISS, Injury Severity Score; GCS, Glasgow Coma Scale; CT, computerized tomography. Mild TBI: admission GCS 13-15; moderate TBI: admission GCS 9-12; severe TBI: admission GCS 3-8. The + sign indicates a mixed lesion on CT. 


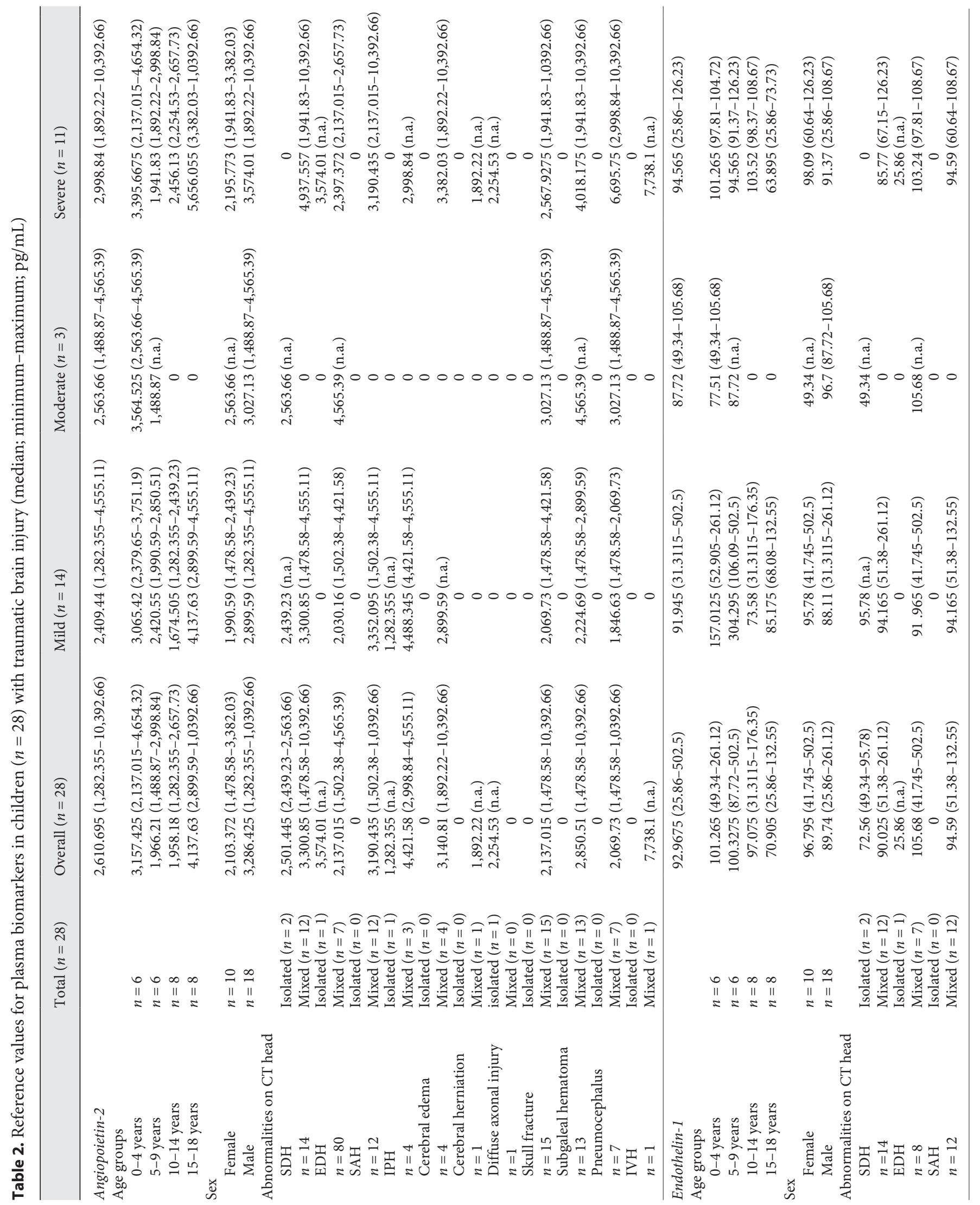




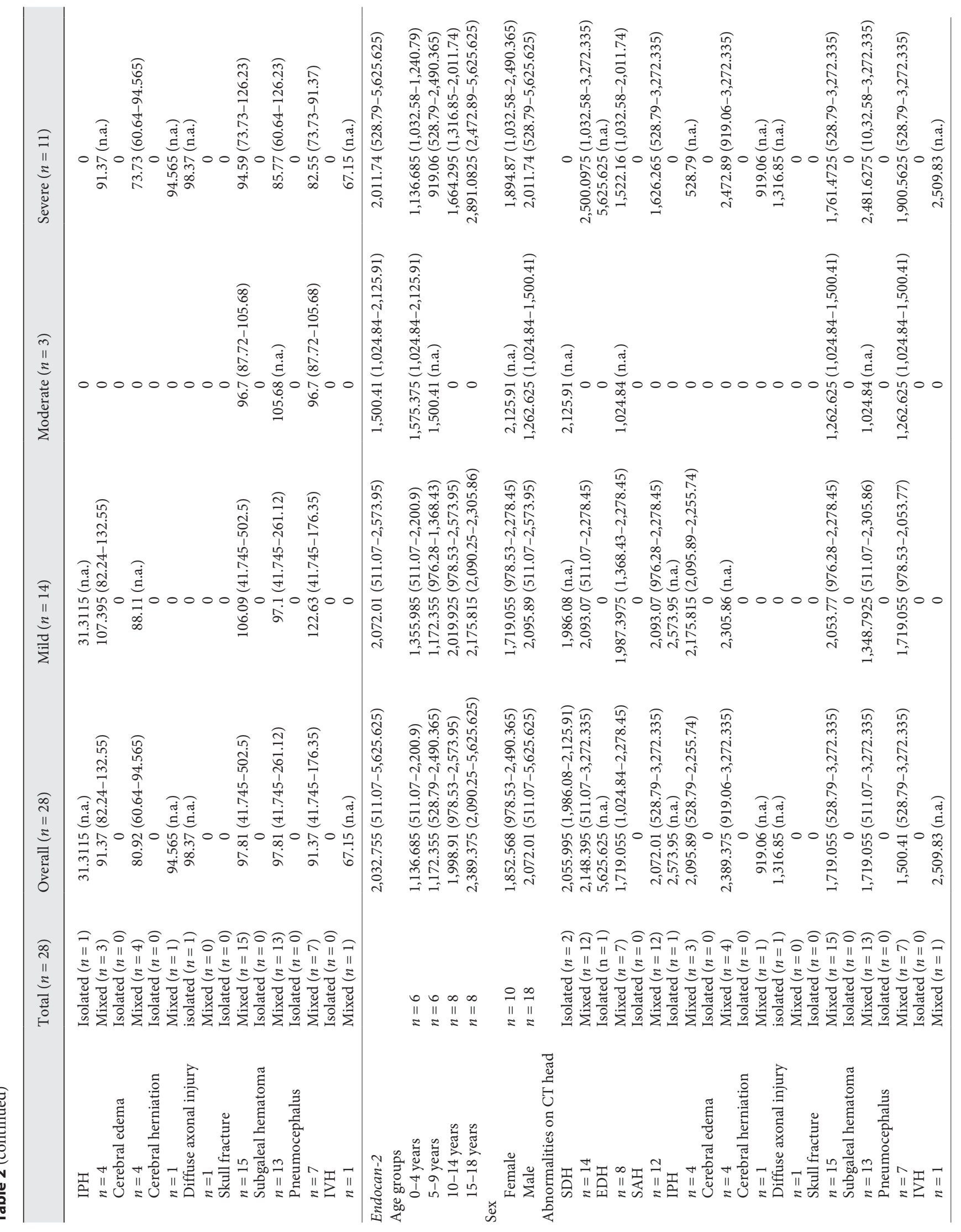




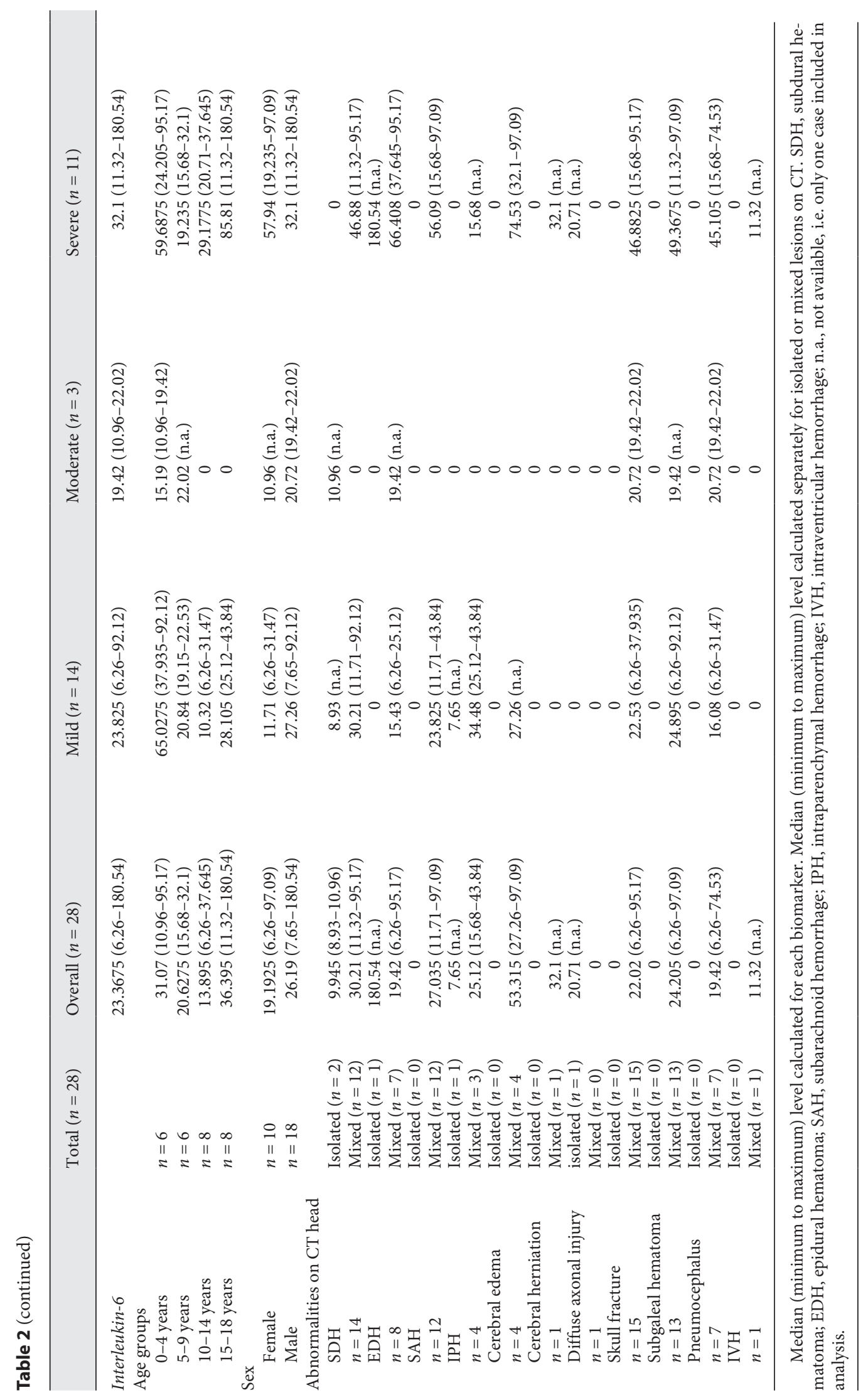


presented in this study have been previously published [33]. The study was approved by the Institutional Review Board at the University of Washington.

\section{Data Collection}

Biomarker Assay

In tandem with venipuncture performed for clinical care, blood was collected for the study into 6-mL vacutainers (BD $\mathrm{K}_{2} \mathrm{EDTA}$, $10.8 \mathrm{mg} /$ tube) and after inverting 10 times, it was immediately placed on ice. Blood samples were then centrifuged at 2,380 r.p.m. for $11 \mathrm{~min}$ within $2 \mathrm{~h}$ of the draw. Plasma was removed from the tube after centrifugal fractionation and aliquoted into $0.5-\mathrm{mL}$ cryovials and frozen at $-80^{\circ} \mathrm{C}$ in a freezer for long-term storage. EMD Millipore's MILLIPLEX MAP assays were used to quantify endocan-1 [34], AP-2, ET-1 [35], and IL-6 [36]. Plasma biomarker levels are expressed in picograms per milliliter.

\section{Clinical Data}

Demographic and clinical data were abstracted from the hospital electronic medical record. Admission head CT imaging reports were reviewed to classify radiographic characteristics of TBI (i.e., subdural hematoma, epidural hematoma, subarachnoid hemorrhage, intraparenchymal hemorrhage, cerebral edema, cerebral herniation, skull fracture, subgaleal hematoma, pneumocephalus, or intraventricular hemorrhage).

TBI versus Other Pediatric Conditions

We reviewed published studies in pediatric TBI, other pediatric disease states, in healthy children and adolescents. Using PubMed, a search was conducted using the following search words: angiopoietin-2, endothelin-1, endocan-2, and interleukin-6. The search was restricted between 1975 and 2018, and to studies including patients between 0 and 18 years of age.

\section{Statistical Analysis}

Based on admission Glasgow Coma Score (GCS), TBI severity was categorized as mild (GCS 13-15), moderate (GCS 9-12), and severe (GCS 3-8). Patients were grouped by age: (1) 0-4 years, (2) 5-9 years, (3) 10-14 years, and (4) 15-18 years. Lesions on head CT were grouped as isolated and mixed (more than 1 lesion). Further, these were divided into extra-axial hemorrhage (subdural/ extradural hematoma), and intra-axial hemorrhage (subarachnoid, intraparenchymal, intraventricular hemorrhage). Details on cerebral autoregulation testing on this cohort have been previously described [33]. Patients who had transcranial Doppler sonography were defined with impaired (autoregulation index $<0.4$ ) and normal cerebral autoregulation (autoregulation index $\geq 0.4$ ).

Since data were not normally distributed, the Wilcoxon ranksum test was used to examine the relationship between the 4 biomarkers (AP-2, ET-1, EC-2, IL-6), as well as the relationship of each biomarker and (a) TBI severity, (b) age groups, (c) head CT lesions, and (4) sex. Individual concentrations and patterns of the relationships between the 4 biomarkers were examined in relation to GCS and Injury Severity Score (ISS) and tested using the nonparametric Spearman's (rho) rank correlation analysis. We examined biomarker concentrations by age group, and in comparison to healthy controls and specific disease types.

$p$ values $<0.05$ were considered significant. Summary data and the relationship between biomarker levels and TBI characteristics are presented as numbers (percent) and medians (interquartile range, IQR). Multivariable linear regression was conducted to examine the impact of impaired autoregulation on levels of each of the 4 biomarkers, adjusting for TBI severity. Data are presented as coefficients with $95 \%$ confidence intervals. Analysis was conducted using Stata 13.1 (College Station, TX, USA) [37].

\section{Results}

\section{Patient Characteristics}

Table 1 summarizes demographics and illness severity of the 28 participants, who were 11 (IQR 5-15.5) years old, mostly male (64.3\%), with ISS 25 (IQR 17-27.10), with median admission GCS 11 (IQR 6-15). Upon hospital admission, 14 children had mild TBI, 3 children had moderate TBI, and 11 children had severe TBI. Overall, the intensive care unit length of stay was 1.9 (IQR 1.1-5.6) days, the hospital length of stay was 6.5 (IQR 1.7-11.5) days, the discharge GCS score was 15 (IQR 15-15). Twenty $(71.4 \%)$ patients were discharged home, 7 (25\%) patients were discharged to an inpatient rehabilitation facility, and 1 (3.6\%) expired.

Participants underwent a total of 64 blood draws between hospital days 1 and 10 as follows: day $1(n=7)$, day $2(n=14)$, day $3(n=13)$, day $4(n=5)$, day $5(n=10)$, day $6(n=5)$, day $7(n=3)$, day $8(n=4)$, day $9(n=2)$, and day $10(n=1)$. Seventeen children had repeat sampling between days 1 and 10 .

\section{Plasma Biomarker Levels}

Overall, Initial, and Temporal Trends

Table 2 shows that for the full cohort of children, median biomarker levels over the first 10 days were: AP-2 (2,610.7; range 1,282.36-10,392.66 pg/mL), ET-1 (92.97; range $25.86-502.5 \mathrm{pg} / \mathrm{mL}), \mathrm{EC}-2$ (2,032.76; range 511.07$5,625.63 \mathrm{pg} / \mathrm{mL})$, and IL-6 (23.37; range $6.26-180.54 \mathrm{pg} /$ $\mathrm{mL})$.

Although there were no statistically significant differences in biomarker expression either between day 1-3 and day 4-10 or between day 1-7 and day 8-10, Figure 2 suggests that compared to mild and moderate TBI, patients with severe TBI have higher levels of AP-2, IL-6, and EC-2 through day 10 after admission.

\section{Patterns in Biomarker Expression}

Clinical Characteristics and Biomarker Expression

Biomarker levels were comparable across the TBI severity spectrum (Fig. 3 ) across all 4 biomarkers. Table 2 shows large variation in median AP-2, median ET-1, median EC2 , and median IL-6 levels within TBI severity groups and no significant difference by TBI severity (Fig. 3). 


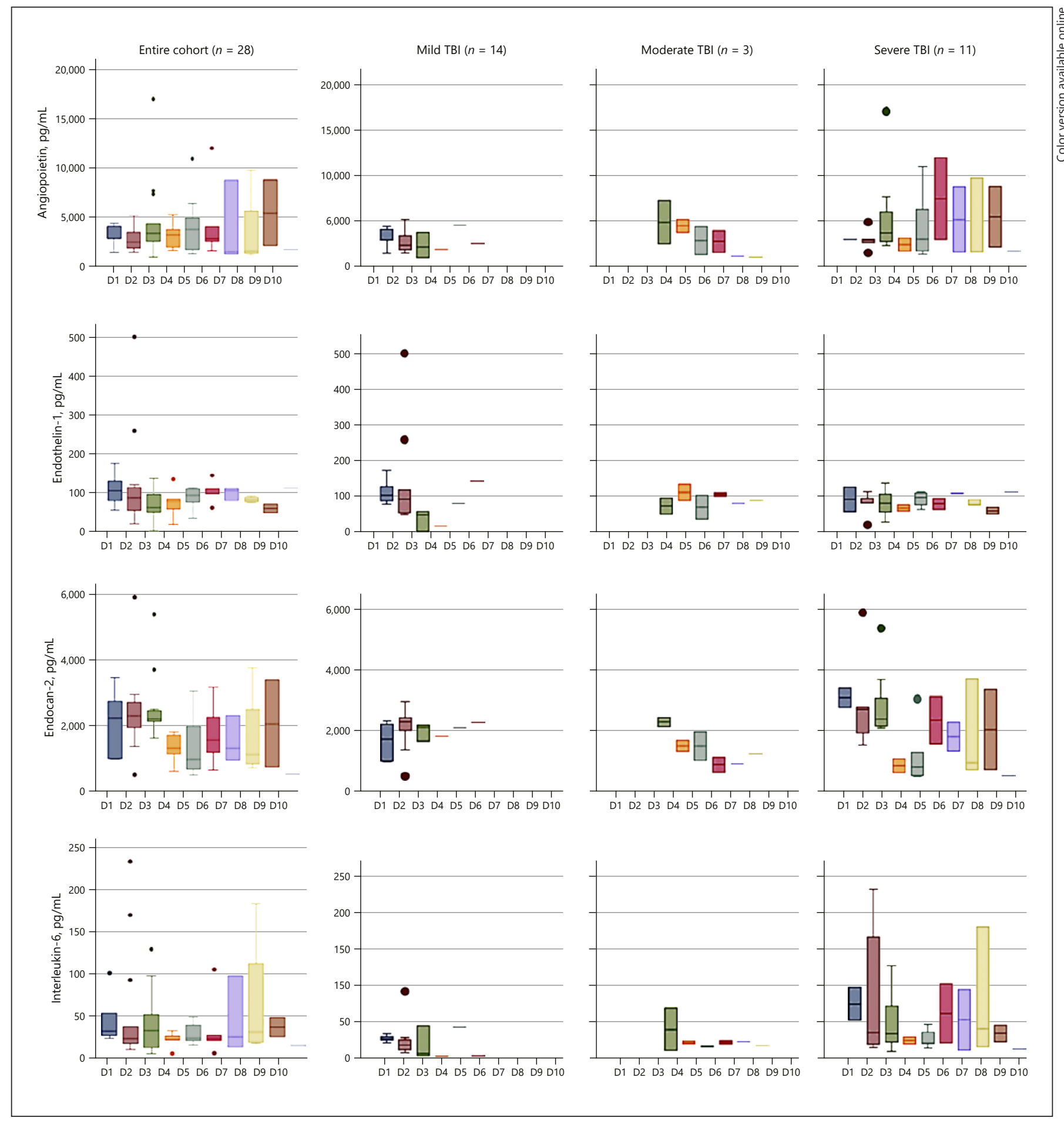

Fig. 2. Patterns of plasma biomarker expression in 28 children by severity of traumatic brain injury (TBI) and by day of admission. Mild TBI, admission Glasgow Coma Score (GCS) 13-15; moderate TBI, admission GCS 9-12; severe TBI, admission GCS 3-8; D1, admission day 1; D10, admission day 10. Data suggest that compared to mild and moderate TBI, patients with severe TBI have higher levels of angiopoietin-2, interleukin-6, and endocan-2 through day 10 after admission. 


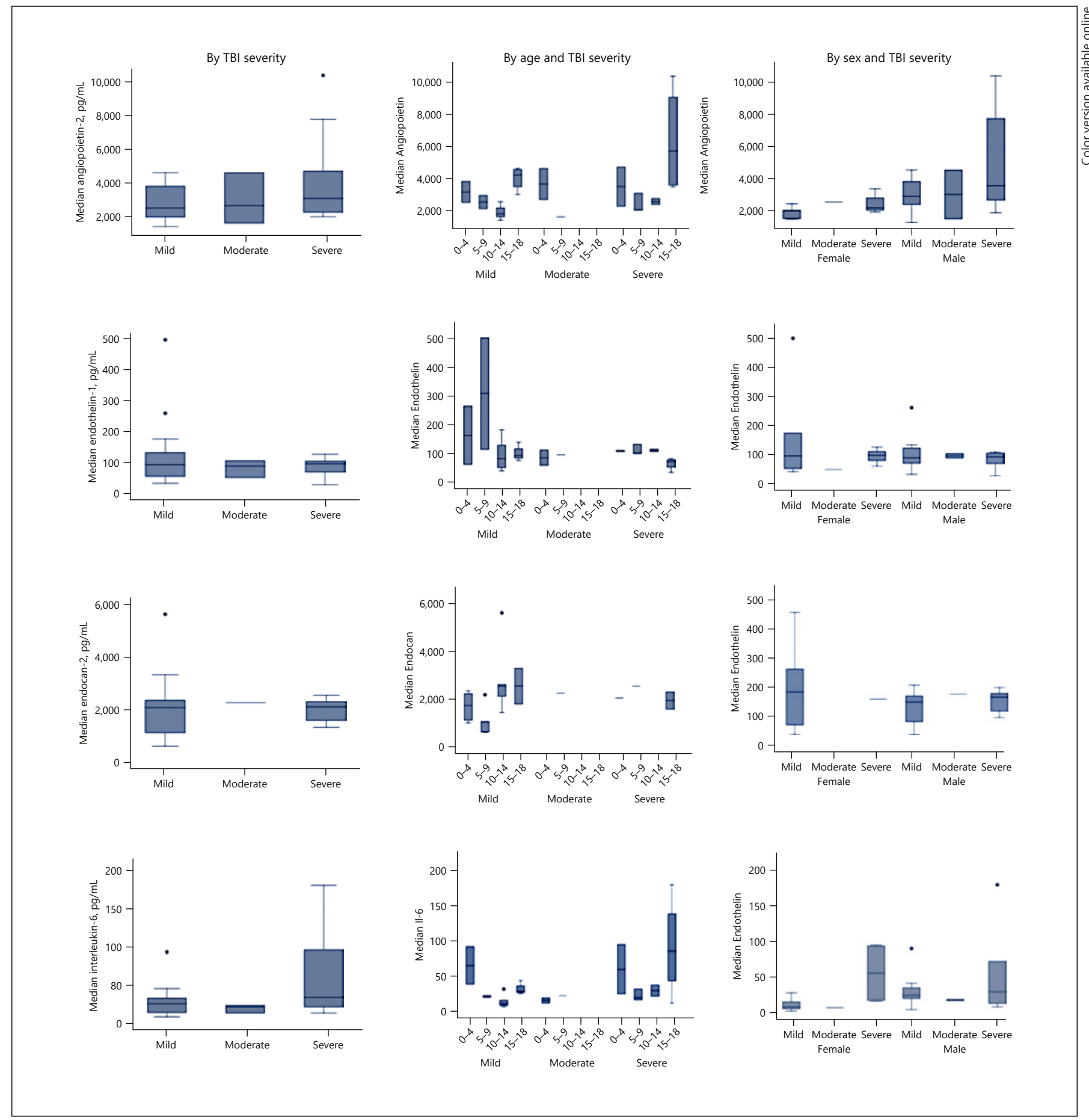

Fig. 3. Patterns of plasma biomarkers in 28 children by traumatic brain injury (TBI) severity, age, and sex. Mild TBI, admission Glasgow Coma Score (GCS) 13-15, $n=11$; moderate TBI, admission GCS 9-12, $n=3$; severe TBI, admission GCS 3-8, $n=14$. Age groups 0-4, 5-9, 10-14, and 15-18 years. Median biomarker levels did not vary significantly by TBI severity, age group, or sex. 
Fig. 4. Patterns of plasma biomarkers in 28 children with traumatic brain injury (TBI) by Glasgow Coma Score (GCS) and Injury Severity Score (ISS). Biomarker patterns suggest an inverse relationship between GCS and angiopoietin-2, GCS and interleukin-6, ISS and endothelin-1, but a direct relationship between GCS and endothelin- 1 and ISS and angiopoietin- 2 .
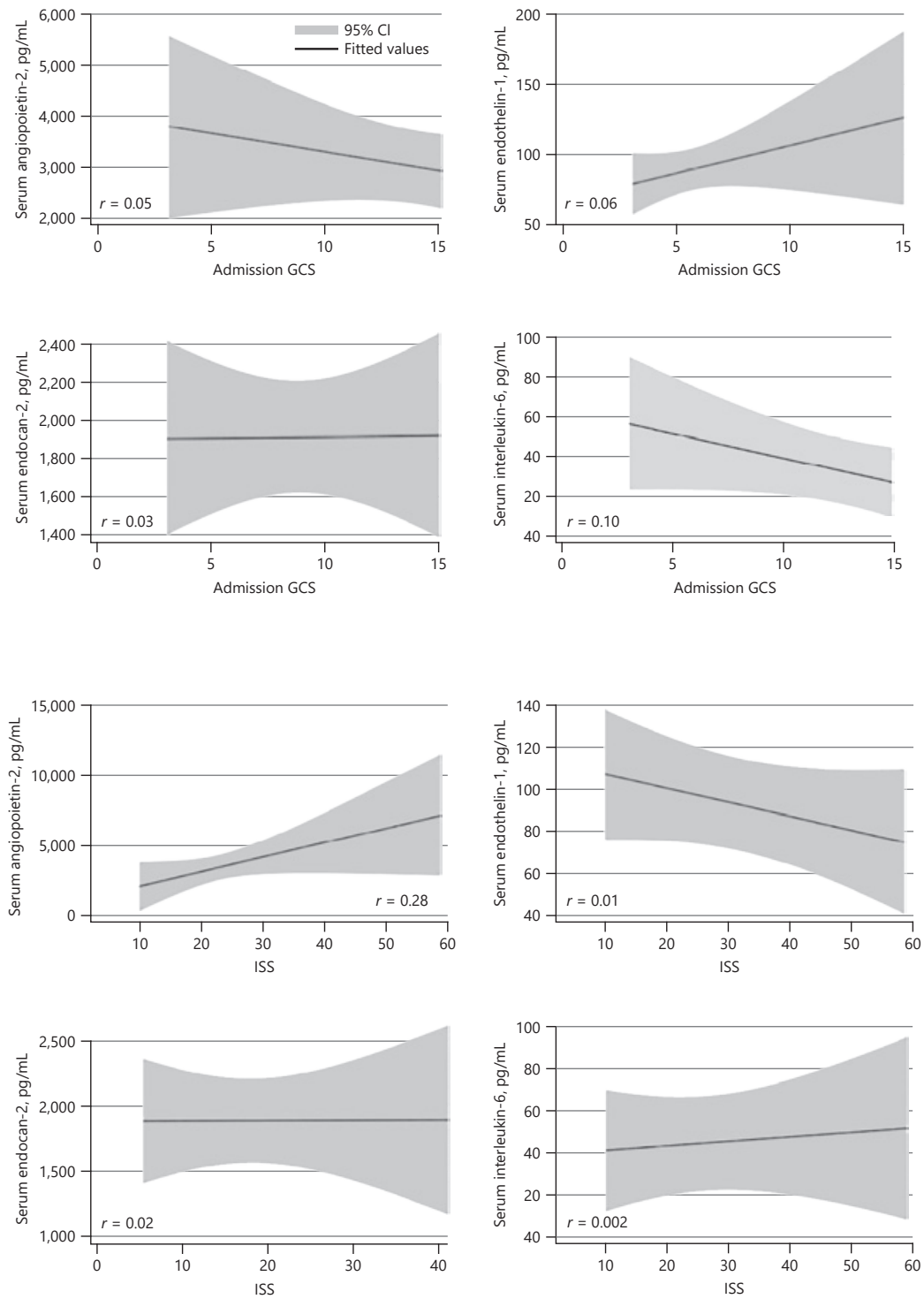

Median biomarker levels did not vary significantly by TBI severity, age group, or sex (Fig. 3; Table 2). However, biomarker patterns suggest an inverse relationship between GCS and AP-2, GCS and IL-6, ISS and ET-1, but a direct relationship between GCS and ET-1 and ISS and AP-2 (Fig. 4). However, none of these relationships were statistically significant.

\section{Biomarker Levels and CT Lesions}

Five (18\%) patients had an isolated lesion on CT: subdural hematoma $(n=2)$, epidural hematoma $(n=1)$, intraparenchymal hemorrhage $(n=1)$, and diffuse axonal injury $(n=1)$. Seventeen patients had extra-axial hemorrhage while 9 patients had extra- and intra-axial hemorrhage. Levels of AP-2, ET-1, EC-2, and IL-6 associated with isolated and mixed CT lesions are presented in Table 2. There was no difference in biomarker levels by head CT lesion type.

\section{Relationship between Plasma Biomarkers}

Biomarker patterns suggest an inverse relationship between AP-2 and ET-1, AP-2 and EC-2, but a direct relationship between AP-2 and IL-6, IL-6 and EC-2, and IL-6 and ET-1 (Fig. 5). 

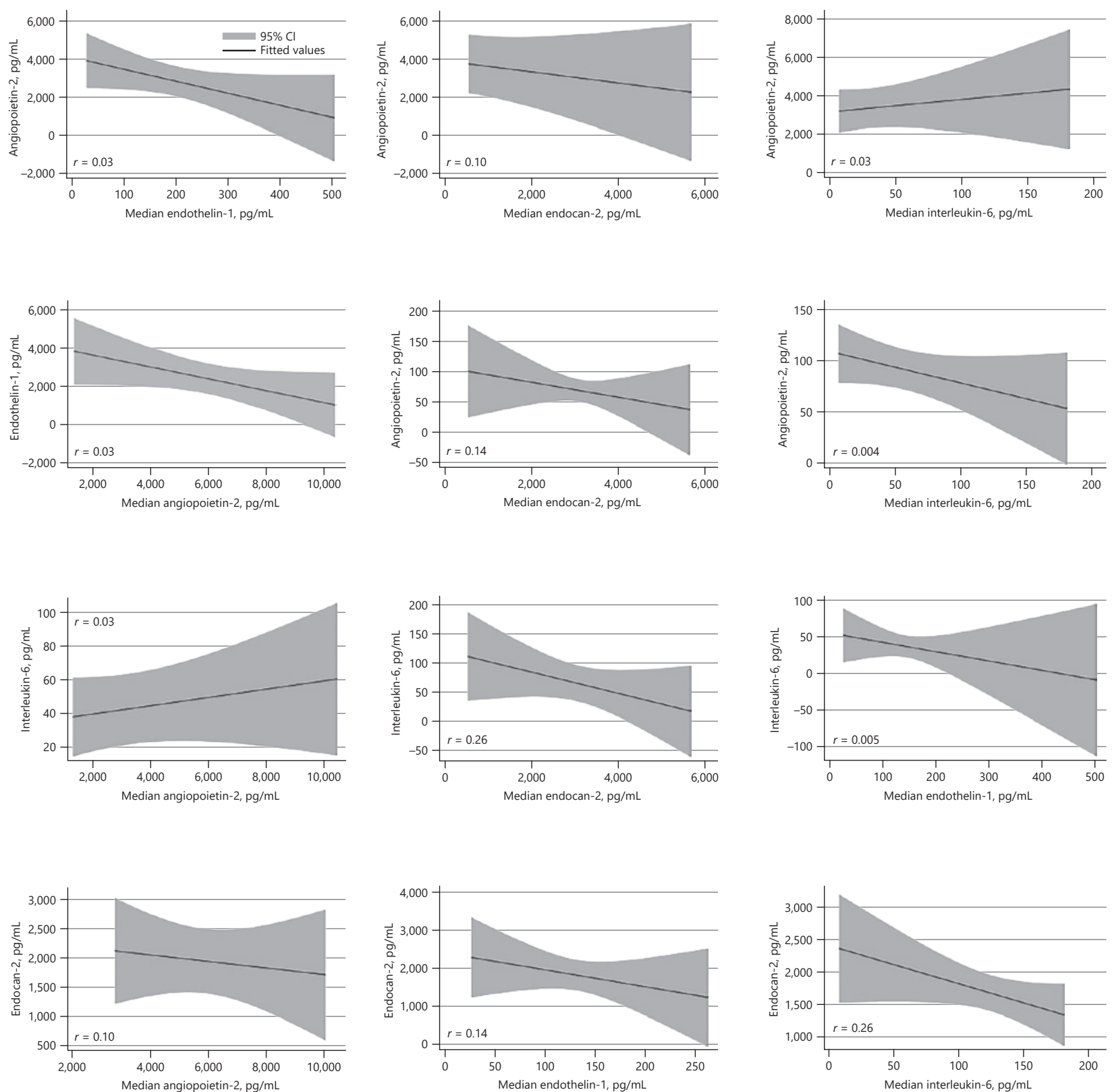

Fig. 5. The interplay between plasma angiopoietin-2, endothelin-1, endocan-2, and interleukin-6. Note: weighted by TBI severity. Biomarker patterns suggest an inverse relationship between angiopoietin- 2 and endothelin-1, angiopoietin- 2 and endocan-2, but a direct relationship between angiopoietin- 2 and interleukin- 6 , interleukin- 6 and endocan-2, and interleukin-6 and endothelin-1.

\section{Association between Plasma Biomarkers and}

\section{Cerebral Autoregulation}

As shown in Table 3, after adjusting for TBI severity, we found no statistically significant association between biomarker expression and cerebral autoregulation status.

Biomarkers of Inflammation and Vascular Homeostasis after Pediatric TBI

\section{Comparison of Biomarker Expression to Other Conditions}

Figure 6 shows an overlap between AP-2, ET-1, and IL-6 biomarker levels and published data from non-TBI cohorts and age-matched controls. No reports of EC-2 levels were found. 


\section{Discussion}

The purpose of this preliminary study was to explore the expression of inflammatory and vascular homeostatic plasma biomarkers in children hospitalized with TBI. While IL- 6 has been studied to a limited extent, this is the first study to report on AP-2, ET-1, and EC-2, and to examine the levels, patterns, and the interplay between these biomarkers in pediatric TBI. Our findings are that: (1) although not statistically significant, levels of inflammatory and vascular homeostatic biomarker expression suggest abnormalities in inflammation and vascular homeostasis during the first 10 days related to TBI severity, (2) patterns of biomarker expression suggest an interplay between inflammation and vascular homeostasis, (3) the variation in biomarker levels within TBI severity, age, and by sex suggests other contributory factors to biomarker expression, and (4) there is no relationship between biomarker expression and cerebral autoregulation status.

Broadly, biomarkers relevant to TBI can be categorized as related to inflammatory (interleukins, marinobufagenin), traumatic neuronal/axonal (tau protein, neurofilaments, neuron-specific enolase [NSE], glial fibrillary acid protein, myelin basic protein [MBP], ubiquitin carboxyl-terminal hydrolase isoenzyme L1]), spectrin breakdown products, neutrophil gelatinase-associated lipocalin, blood-brain barrier integrity (angiopoietin-1, AP -2 [15], cerebrospinal fluid/plasma albumin ratio, tight junction proteins, NSE, S100B, GPAP), and genetic biomarkers such as apolipoprotein-E and brain-derived neurotrophic factor [9].

We targeted the 4 biomarkers to examine inflammatory and vascular homeostatic pathways because of prior work showing alterations in these pathways and because these complex secondary TBI pathways may be related and/or differentially expressed in pediatric TBI. Results from this study imply that EC-2 may not be expressed and be as representative of vascular homeostatic processes as perhaps AP-2 and ET-1 but these results need to be further confirmed.

Although not statistically significant, this study suggests that compared to mild TBI, there may be greater upregulation of inflammation and vascular homeostatic processes in severe TBI. A prior study on children with TBI by Chiaretti et al. [7] reported increases in plasma IL-6 levels with worsening TBI severity. The pattern similarities between AP- 2 and IL- 6 suggest that both inflammation and blood-brain-barrier breakdown occur with worsening TBI severity, and AP-2 may be considered as a surrogate marker of injury severity, similar to previous studies reporting higher levels of AP-2 in septic shock compared to sepsis [38, 39], and as reported with IL-6 [4]. Present results imply that AP-2 and IL- 6 may also be surrogate markers of pediatric TBI severity, but this will require confirmation in a larger cohort of children with pediatric TBI. Despite the lack of significance, the present

Table 3. Association between plasma biomarkers and cerebral autoregulation in hospitalized children $(n=25)$ with traumatic brain injury

\begin{tabular}{|c|c|c|c|c|}
\hline & $\begin{array}{l}\text { Angiopoietin-2 } \\
\text { coefficient }(95 \% \mathrm{CI})\end{array}$ & $\begin{array}{l}\text { Endothelin-1 } \\
\text { coefficient }(95 \% \text { CI })\end{array}$ & $\begin{array}{l}\text { Endocan-2 } \\
\text { coefficient }(95 \% \mathrm{CI})\end{array}$ & $\begin{array}{l}\text { Interleukin-6 } \\
\text { coefficient }(95 \% \mathrm{CI})\end{array}$ \\
\hline \multicolumn{5}{|c|}{ Cerebral autoregulation } \\
\hline Impaired & $350.63(-1,498.11$ to $2,199.38)$ & $-31.02(-118.62$ to 56.57$)$ & $-316.66(-1,304.84$ to 671.51$)$ & $-13.29(-48.98$ to 22.39$)$ \\
\hline \multicolumn{5}{|c|}{ TBI severity } \\
\hline Mild & Reference & Reference & Reference & Reference \\
\hline Severe & $1,271.21(-577.53$ to $3,119.95)$ & $-37.29(-124.89$ to 50.3$)$ & $445.06(-543.12$ to $1,433.24)$ & $32.92(-2.76$ to 68.6$)$ \\
\hline
\end{tabular}

Values expressed as picograms per milliliter.

Fig. 6. Comparison of median plasma biomarker levels with prior work in traumatic brain injury (TBI) and non-TBI cohorts. a Plasma angiopoietin-2 comparison studies. ${ }^{*}$ Shroff et al. [42], 2013. ** Giuliano et al. [38], 2007. CKD, chronic kidney disease; SIRS, systemic inflammatory response syndrome. b Plasma endothelin-1 comparison studies. * Kageyama et al. [43], 2007. ** Bakr et al. [44], 2002. *** El-Gamal et al. [45], 2002. CPB, cardiopulmonary bypass. c Plasma interleukin -6 comparison study. ${ }^{*}$ Chiaretti et al. [1], 2005. TBI, traumatic brain injury. In the present study, numbers of patients per age group were: $0-4$ years, $n=6 ; 5-9$ years, $n=6$; $10-14$ years, $n=8 ; 15-19$ years, $n=8$.

(For figure see next page.) 

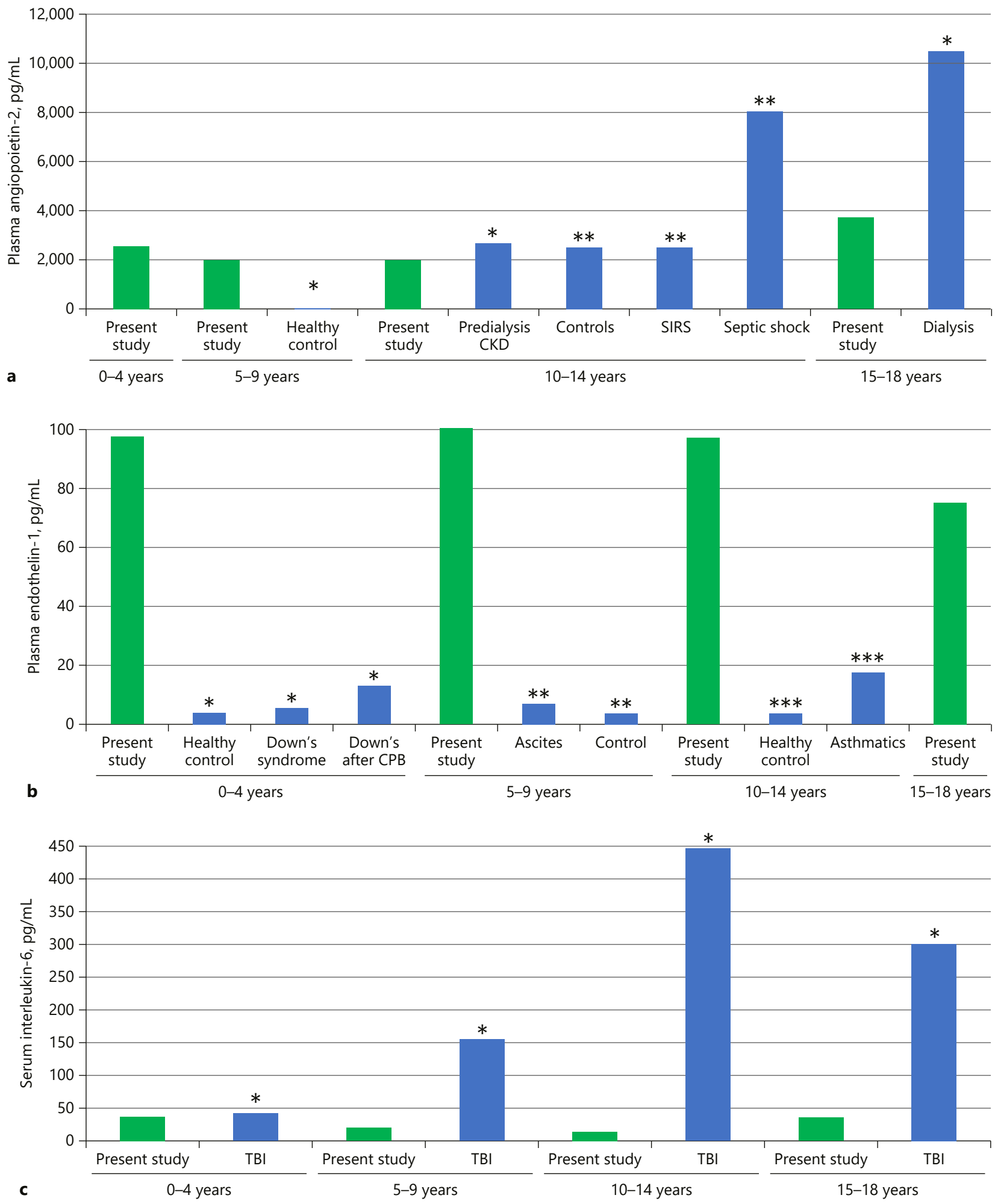
study is the first to report on the relationship between AP-2, ET-1, EC-2 by and across TBI severity.

The results of this study suggest no relationship between levels of AP-2 and IL- 6 with TBI severity, age, and sex. However, age-related increases in NSE, S100B, and MBP have been described previously [40], and we cannot exclude an age-dependent increase in biomarker levels due to an age-dependent increase in blood-brain barrier permeability. Prior work by van Engelen et al. [40] examining S100B, NSE, and MBP in a study of 79 specimens of cerebrospinal fluid showed no sex differences in TBI, and there is no study examining the relationship with IL-6 [7]. Present results suggest that interpretation of plasma biomarker data in pediatric TBI may be incomplete without knowledge of age and sex of the population studied. Since clinical outcomes after pediatric TBI may be influenced by age and sex [41], future studies should validate our findings in a larger population of children with TBI that includes sufficient numbers of males, females, and age groups. We speculate that inflammatory and vascular homeostatic processes extend beyond the initial critical period after severe pediatric TBI (i.e., the first $72 \mathrm{~h}$ ), implying that these children may continue to remain at high risk for secondary brain insults from these processes beyond the first week after injury.

Mechanistically, IL-6 is an inflammatory biomarker, and AP-2 and ET-1 are markers of blood-brain barrier breakdown [15] and endothelial dysfunction [12], respectively. Thus, the interplay between these biomarkers deserves some discussion. Whether the biomarkers follow direct or inverse relationships, and whether and how they interact to impact cerebral autoregulation is unknown. Future work is needed to confirm these biomarker relationships and effects.

\section{Limitations}

This preliminary study has some limitations. Although we examined biomarker expression across the TBI sever- ity spectrum for initial assessment, we did not collect data from age-matched healthy controls. Due to the small sample size, our data are primarily descriptive, and although children in the moderate TBI group are very few, we felt it important to present biomarker levels for this group of patients separately. Despite these limitations, we provide new data on patterns of plasma biomarker expression reflecting inflammation and vascular homeostasis in children hospitalized with TBI.

\section{Conclusions}

Plasma concentrations of inflammatory and vascular homeostatic biomarkers suggest a role for inflammation and disruption of vascular homeostasis during the first 10 days across the severity spectrum of pediatric TBI. Although not statistically significant, and without impact on cerebral autoregulation, biomarker patterns suggest a relationship between inflammation and alterations in vascular homeostasis. The large variation in biomarker levels within TBI severity and age groups, and by sex suggests other contributory factors to biomarker expression.

\section{Statement of Ethics}

The study was approved by the Institutional Review Board at the University of Washington.

\section{Disclosure Statement}

The authors report no conflict of interest relevant to this study.

\section{Funding Sources}

National Institutes of Neurological Diseases and Stroke (R21NS095321-02: Vavilala).

\section{References}

1 Chiaretti A, Genovese O, Aloe L, Antonelli A, Piastra M, Polidori G, et al. Interleukin 1beta and interleukin 6 relationship with paediatric head trauma severity and outcome. Childs Nerv Syst. 2005 Mar;21(3): 185-93.

2 Stanley RM, Bonsu BK, Zhao W, Ehrlich PF, Rogers AJ, Xiang H. US estimates of hospitalized children with severe traumatic brain injury: implications for clinical trials. Pediatrics. 2012 Jan;129(1):e24-30.
3 Schneier AJ, Shields BJ, Hostetler SG, Xiang $\mathrm{H}$, Smith GA. Incidence of pediatric traumatic brain injury and associated hospital resource utilization in the United States. Pediatrics. 2006 Aug;118(2):483-92.

4 Chiaretti A, Antonelli A, Mastrangelo A, Pezzotti P, Tortorolo L, Tosi F, et al. Interleukin- 6 and nerve growth factor upregulation correlates with improved outcome in children with severe traumatic brain injury. $J$ Neurotrauma. 2008 Mar;25(3):225-34. 
5 Beers SR, Berger RP, Adelson PD. Neurocognitive outcome and serum biomarkers in inflicted versus non-inflicted traumatic brain injury in young children. J Neurotrauma. 2007 Jan;24(1):97-105.

6 Berger RP, Beers SR, Richichi R, Wiesman D, Adelson PD. Serum biomarker concentrations and outcome after pediatric traumatic brain injury. J Neurotrauma. 2007 Dec; 24(12):1793-801.

7 Chiaretti A, Genovese O, Aloe L, Antonelli A, Piastra M, Polidori G, et al. Interleukin 1beta and interleukin 6 relationship with paediatric head trauma severity and outcome. Childs Nerv Syst. 2005 Mar;21(3):185-93; discussion 194.

8 Lo TY, Jones PA, Minns RA. Combining coma score and serum biomarker levels to predict unfavorable outcome following childhood brain trauma. J Neurotrauma. 2010 Dec; 27(12):2139-45.

9 Kim HJ, Tsao JW, Stanfill AG. The current state of biomarkers of mild traumatic brain injury. JCI Insight. 2018 Jan;3(1):97105.

10 Maisonpierre PC, Suri C, Jones PF, Bartunkova S, Wiegand SJ, Radziejewski C, et al. Angiopoietin-2, a natural antagonist for Tie2 that disrupts in vivo angiogenesis. Science. 1997 Jul;277(5322):55-60.

11 Liu XW, Ma T, Liu W, Cai Q, Wang L, Song HW, et al. Sustained increase in angiopoietin-2, heparin-binding protein, and procalcitonin is associated with severe sepsis. J Crit Care. 2018 Jun;45:14-9.

12 Gozal D, Khalyfa A, Qiao Z, Smith DL, Philby MF, Koren D, et al. Angiopoietin-2 and soluble Tie-2 receptor plasma levels in children with obstructive sleep apnea and obesity. Obesity (Silver Spring). 2017 Jun;25(6):108390.

13 Zan L, Wu H, Jiang J, Zhao S, Song Y, Teng G, et al. Temporal profile of Src, SSeCKS, and angiogenic factors after focal cerebral ischemia: correlations with angiogenesis and cerebral edema. Neurochem Int. 2011 Jul;58(8):872-9.

14 Schönmann SM, Iyer J, Laeng H, Gerber HA, Käser H, Blaser K. Production and characterization of monoclonal antibodies against human neuroblastoma. Int J Cancer. 1986 Feb; 37(2):255-62.

15 Chittiboina P, Ganta V, Monceaux CP, Scott LK, Nanda A, Alexander JS. Angiopoietins as promising biomarkers and potential therapeutic targets in brain injury. Pathophysiology. 2013 Feb;20(1):15-21.

16 Fujimura M, Joo JY, Kim JS, Hatta M, Yokoyama Y, Tominaga T. Preventive Effect of Clazosentan against Cerebral Vasospasm after Clipping Surgery for Aneurysmal Subarachnoid Hemorrhage in Japanese and Korean Patients. Cerebrovasc Dis. 2017;44(1-2): 59-67.

17 Ozawa T, Sugiyama S, Tanaka M, Hattori K. Mitochondrial DNA mutations and disturbances of energy metabolism in myocardium. Jpn Circ J. 1991 Nov;55(11):1158-64.
18 Rong WL, Xiao X, Zhao JL, Yang XW, Hu ZL, Li MH. Different Doses of Clazosentan for Aneurismal Subarachnoid Hemorrhage: A Meta-Analysis of Randomized Controlled Trials. Am J Ther. 2018 Nov/Dec;25(6):e65260.

19 Huseynova SA, Panakhova NF, Hajiyeva AS, Orujova PA, Mukhtarova SN, Agayeva GT. Endothelial dysfunction and developmental outcomes of very low birth weight newborns with hypoxic encephalopathy. J Pak Med Assoc. 2017 Dec;67(12):1857-63.

$20 \mathrm{Gu}$ M, Kawoos U, McCarron R, Chavko M. Protection against Blast-Induced Traumatic Brain Injury by Increase in Brain Volume. BioMed Res Int. 2017;2017:2075463.

21 Spray S, Johansson SE, Radziwon-Balicka A, Haanes KA, Warfvinge K, Povlsen GK, et al. Enhanced contractility of intraparenchymal arterioles after global cerebral ischaemia in rat - new insights into the development of delayed cerebral hypoperfusion. Acta Physiol (Oxf). 2017 Aug;220(4):417-31.

22 Hung VK, Yeung PK, Lai AK, Ho MC, Lo AC, Chan KC, et al. Selective astrocytic endothelin-1 overexpression contributes to dementia associated with ischemic stroke by exaggerating astrocyte-derived amyloid secretion. J Cereb Blood Flow Metab. 2015 Oct; 35(10):1687-96.

23 Chang X, Bian Y, Wu Y, Huang Y, Wang K, Duan T. Endocan of the maternal placenta tissue is increased in pre-eclampsia. Int $]$ Clin Exp Pathol. 2015 Nov;8(11):14733-40.

24 Hentschke MR, Lucas LS, Mistry HD, Pinheiro da Costa BE, Poli-de-Figueiredo CE. Endocan-1 concentrations in maternal and fetal plasma and placentae in pre-eclampsia in the third trimester of pregnancy. Cytokine. 2015 Jul;74(1):152-6.

25 Jiang L, Hu Y, He X, Lv Q, Wang TH, Xia QJ. Breviscapine reduces neuronal injury caused by traumatic brain injury insult: partly associated with suppression of interleukin- 6 expression. Neural Regen Res. 2017 Jan;12(1): 90-5.

26 Jiang L, Xia QJ, Dong XJ, Hu Y, Chen ZW, Chen K, et al. Neuroprotective effect of breviscapine on traumatic brain injury in rats associated with the inhibition of GSK3 $\beta$ signaling pathway. Brain Res. 2017 Apr;1660: $1-9$.

27 Wagner N, Akbarpour A, Mörs K, Voth M, Störmann P, Auner B, et al. Alcohol Intoxication Reduces Systemic Interleukin-6 Levels and Leukocyte Counts After Severe TBI Compared With Not Intoxicated TBI Patients. Shock. 2016 Sep;46(3):261-9.

28 Kumar RG, Diamond ML, Boles JA, Berger RP, Tisherman SA, Kochanek PM, et al. Acute CSF interleukin-6 trajectories after TBI: associations with neuroinflammation, polytrauma, and outcome. Brain Behav Immun. 2015 Mar;45:253-62.
29 Amick JE, Yandora KA, Bell MJ, Wisniewski SR, Adelson PD, Carcillo JA, et al. The Th1 versus Th2 cytokine profile in cerebrospinal fluid after severe traumatic brain injury in infants and children. Pediatr Crit Care Med. $2001 \mathrm{Jul} ; 2(3): 260-4$.

30 Berger RP, Ta'asan S, Rand A, Lokshin A, Kochanek P. Multiplex assessment of serum biomarker concentrations in well-appearing children with inflicted traumatic brain injury. Pediatr Res. 2009 Jan;65(1):97-102.

31 Park SH, Hwang SK. Prognostic Value of Serum Levels of S100 Calcium-Binding Protein B, Neuron-Specific Enolase, and Interleukin-6 in Pediatric Patients with Traumatic Brain Injury. World Neurosurg. 2018 Oct; 118:e534-42.

32 Armstead WM, Riley J, Vavilala MS. TBI sex dependently upregulates ET-1 to impair autoregulation, which is aggravated by phenylephrine in males but is abrogated in females. J Neurotrauma. 2012 May;29(7):1483-90.

33 Lele AV, Watanitanon A, Lakireddy V, Clark-Bell C, Moore A, Zimmerman JJ, et al. Prevalence, Evolution, and Extent of Impaired Cerebral Autoregulation in Children Hospitalized With Complex Mild Traumatic Brain Injury. Pediatr Crit Care Med. 2019 Apr;20(4):372-8.

34 Millipore Sigma. Millipex MAP Human Cardiovascular Disease(CVD) Magnetic Bead Panel 1: Merck KGaA; 2018. Available from: http://www.emdmillipore.com/US/en/ product/MILLIPLEX-MAP-Human-Cardiovascular-Disease-CVD-Magnetic-BeadPanel-1-Cardiovascular-Disease-MultiplexAssay,MM_NF-HCVD1MAG-67K.

35 Millipore Sigma. Millipex MAP Human Angiogenesis/Growth Factor Magnetic bead Panel: Merck GaA; 2018. Available from: http://www.emdmillipore.com/US/en/ product/MILLIPLEX-MAP-Human-Angiogenesis/Growth-Factor-Magnetic-BeadPanel-Cancer-Multiplex-Assay,MM_NFHAGP1MAG-12K.

36 Millipore Sigma. Millipex MAP Human Cytokin/Chemokine Magnetic Bead Panel: Merck KGaA; 2018. Available from: http:// www.emdmillipore.com/US/en/product/ MILLIPLEX-MAP-Human-Cytokine/Chemokine-Magnetic-Bead-Panel-Immunology-Multiplex-Assay,MM_NF-HCYTOMAG-60K.

37 StataCorp. Stata/IC. 15.1 ed. College Station (TX): StataCorp LP; 2017.

38 Giuliano JS Jr, Lahni PM, Harmon K, Wong HR, Doughty LA, Carcillo JA, et al. Admission angiopoietin levels in children with septic shock. Shock. 2007 Dec;28(6):650-4.

39 Giuliano JS Jr, Tran K, Li FY, Shabanova V, Tala JA, Bhandari V. The temporal kinetics of circulating angiopoietin levels in children with sepsis. Pediatr Crit Care Med. 2014 Jan; 15(1):e1-8. 
40 van Engelen BG, Lamers KJ, Gabreels FJ, Wevers RA, van Geel WJ, Borm GF. Age-related changes of neuron-specific enolase, S-100 protein, and myelin basic protein concentrations in cerebrospinal fluid. Clin Chem. 1992 Jun;38(6):813-6.

41 Bazarian JJ, Blyth B, Mookerjee S, He H, McDermott MP. Sex differences in outcome after mild traumatic brain injury. J Neurotrauma. 2010 Mar;27(3):527-39.
42 Shroff RC, Price KL, Kolatsi-Joannou M, Todd AF, Wells D, Deanfield J, Johnson RJ, Rees L, Woolf AS, Long DA. Circulating angiopoietin-2 is a marker for early cardiovascular disease in children on chronic dialysis. PLoS One. 2013;8(2):e56273.

43 Kageyama K, Hashimoto S, Nakajima Y, Shime N, Hashimoto S. The change of plasma endothelin-1 levels before and after surgery with or without Down syndrome. Paediatr Anaesth. 2007 Nov;17(11):1071-7.
44 Bakr AM, Abdalla AF, El-Marsafawy H, AbuHashem I, El-Regal ME, Amer T, Abdel-Khalik MK, Mostafa H, A-Kader HH. Plasma endothelin-1 concentrations in children with cirrhosis and their relationship to renal function and the severity of portal hypertension. J Pediatr Gastroenterol Nutr. 2002 Aug;35(2):149-53.

45 El-Gamal Y, Hossny E, Awwad K, Mabrouk $\mathrm{R}$, Boseila N. Plasma endothelin-1 immunoreactivity in asthmatic children. Ann Allergy Asthma Immunol. 2002 Apr;88(4):370-3. 